\title{
Convergence of a stabilized discontinuous Galerkin method for incompressible nonlinear elasticity
}

\author{
Davide Baroli • Alfio Quarteroni • \\ Ricardo Ruiz-Baier
}

Received: 28 December 2011 / Accepted: 5 November 2012 /

Published online: 16 December 2012

(C) Springer Science+Business Media New York 2012

\begin{abstract}
In this paper we present a discontinuous Galerkin method applied to incompressible nonlinear elastostatics in a total Lagrangian deformationpressure formulation, for which a suitable interior penalty stabilization is applied. We prove that the proposed discrete formulation for the linearized problem is well-posed, asymptotically consistent and that it converges to the corresponding weak solution. The derived convergence rates are optimal and further confirmed by a set of numerical examples in two and three spatial dimensions.
\end{abstract}

Communicated by: Zhongying Chen.

Dedicated to Prof. Wolfgang L. Wendland in occasion of his 75th birthday.

D. Baroli

Dipartimento di Fisica e Matematica, Università degli studi dell'Insubria,

Via Valleggio 11, 22100 Como, Italy

e-mail: davide.baroli@epfl.ch

A. Quarteroni · R. Ruiz-Baier (四)

CMCS-MATHICSE-SB, Ecole Polytechnique Fédérale de Lausanne,

1015 Lausanne, Switzerland

e-mail: ricardo.ruiz@epfl.ch

A. Quarteroni

e-mail: alfio.quarteroni@epfl.ch

A. Quarteroni

MOX - Modellistica e Calcolo Scientifico, Dipartimento di Matematica,

Politecnico di Milano, 20133 Milano, Italy

Present Address:

D. Baroli

MOX - Modellistica e Calcolo Scientifico, Dipartimento di Matematica,

Politecnico di Milano, 20133 Milano, Italy 
Keywords Nonlinear elasticity • Discontinuous Galerkin formulation • Incompressible material $\cdot$ Edge-based stabilization

Mathematics Subject Classifications (2010) 65 N30 • 65N12 • 74B20

\section{Introduction}

The numerical simulation of problems arising in finite elastostatics entails the resolution of nontrivial quasilinear elliptic partial differential equations (PDEs) accounting for the balance of forces and related constitutive laws. Nonlinear elasticity problems have a large number of applications in structural analysis, biomechanics and engineering design. In particular, our development is motivated by the study of anisotropic soft living materials such as the heart tissue, which presents the ability of actively deform without the need of external loads $[13,14,19]$. If the assumption of incompressibility of the underlying material is considered, and under a Lagrangian formulation, the momentum equation is coupled to a nonlinear volumetric constraint (the determinant of the deformation gradient must be equal to 1). In contrast, the usual divergence free condition is employed in fluid mechanics, linear elasticity, and other systems formulated in the current (deformed) configuration.

Finite elements are by far the most used strategy for the spatial discretization of elasticity problems. This because of their flexibility and computational robustness combined with their sound mathematical foundations that allow the derivation of rigorous error bounds. Unfortunately there is no such thing as "the correct choice" for the particular type of finite element discretization. Many effects need to be considered, specially for large deformation elasticity problems, and slightly different methods may feature substantial differences depending on the choice of finite element spaces, hexahedra or tetrahedra, conforming or nonconforming, formulations leading to symmetric or nonsymmetric linear systems, and so on. In addition, it is well known that for low-order finite element approximations of elasticity problems subject to the volume preserving constraint, the undesirable so-called volume locking phenomenon, in which the displacement decreases severely in a non-physical fashion may show up [12]. Several remedies have been proposed in the literature, including for instance resorting to different kinds of mixed and double-mixed formulations $[4,6,10,15]$. Herein, following the framework in [5, 27], we formulate a suitable discontinuous Galerkin (DG) method. In particular, in its interior penalty variant [3], to weakly impose interelement continuity, the inclusion of penalty terms in the energy functional is a strategy to reduce the spurious numerical instabilities generated by a discontinuous formulation. These terms can be least squares contributions arising from, e.g., constitutive equations or jump penalty terms. Further well known advantages of DG formulations include the ability of handling complex domains, anisotropic and nonconforming meshes, PDEs with discontinuous coefficients, variable approximation degrees, featuring better accuracy than classical finite elements 
for a given number of degrees of freedom, and so on (see [1, 8] and the references therein).

A common approach to solve problems in finite elasticity consists in employing an iterative Newton method, which requires a consistent linearization of the original weak formulation. Moreover in each iteration the linearized problem has to fulfill, at least for sufficiently small deformations, solvability and stability in the sense of the classical Babuška-Brezzi theory for saddlepoint problems [6].

In this paper we propose, analyze, and implement an interior penalty stabilized discontinuous Galerkin method for a deformation-pressure formulation of a nonlinear elasticity problem arising in the study of soft living materials [14]. The deformations and pressure field are discretized with piecewise polynomials of maximal degrees $k$ and $k-1$, respectively. The error analysis we carry out shows that the approximate deformations in the energy norm, and pressure in the $L^{2}-$ norm, converge with optimal orders $\mathcal{O}\left(h^{k}\right)$ and $\mathcal{O}\left(h^{k+1}\right)$, respectively. The novelty of the present paper lies in the convergence study of the DG formulation for nonlinear incompressible materials. Similar approaches to the one developed herein can be found in e.g. [20], where the authors present a DG method for compressible hyperelastic materials, based on a Hu-Washizu-de Veubeke principle and analyze its consistency and linearized stability, or the convergence analysis presented in the general framework of [21]. For a complete analysis of stabilized DG methods to general nonlinear elasticity, we refer to [25].

The rest of the paper is organized as follows. Section 2 contains the description of a boundary value problem in nonlinear elasticity, followed by an analysis of solvability and stability for a linearized system. The main tools needed for the formulation and analysis of the discrete problem, along with the detailed derivation of error estimates are provided in Section 3. Some numerical tests confirming the predicted convergence rates are given in Section 4, and we close in Section 5 with some conclusions and discussion.

\section{Governing equations of finite elasticity}

Let $\Omega_{o} \subset \mathbb{R}^{d}, d \in\{2,3\}$ denote an elastic body with polygonal boundary $\partial \Omega_{o}$, regarded in its undeformed reference configuration. A point in $\Omega_{o}$ is denoted by $\boldsymbol{x}$, while $\hat{\boldsymbol{u}}: \Omega_{o} \rightarrow \mathbb{R}^{d}$ denotes the deformation field that provides its position in the current configuration.

The tensor $\hat{\mathbf{F}}:=\nabla \hat{\boldsymbol{u}}$ is the gradient of the deformation map. We are interested in the case where the material is (fully) incompressible. This is represented by the constraint $\operatorname{det}(\hat{\mathbf{F}})=1$ that is enforced by the use of a Lagrange multiplier $p$, interpreted as pressure. The total potential energy of the deformed domain in the absence of volume forces is

$$
I(\hat{\boldsymbol{u}}, \hat{p})=\int_{\Omega_{o}} \mathcal{W}(\hat{\boldsymbol{u}}, \hat{p}) d \boldsymbol{x}-\int_{\Gamma_{\mathrm{N}}} \boldsymbol{t} \cdot \hat{\boldsymbol{u}} d s,
$$


for a surface traction $t$ applied on the Neumann boundary $\Gamma_{\mathrm{N}} \subset \partial \Omega_{o}$. Here $\hat{\boldsymbol{u}}, \hat{p}$ represent virtual (admissible) displacements and pressures, respectively. By $\Gamma_{\mathrm{D}}$ we denote the part of the boundary where Dirichlet conditions are applied on the deformations $\left(\left.\hat{\boldsymbol{u}}\right|_{\Gamma_{\mathrm{D}}}=\boldsymbol{g}\right)$, and we assume that $\partial \Omega_{o}=\Gamma_{\mathrm{N}} \cup \Gamma_{\mathrm{D}}$. The function $\mathcal{W}$ in (1) represents a strain stored energy, whose specific form depends on the material properties of the body.

The classical nonlinear elasticity problem consists in finding admissible deformations $\hat{\boldsymbol{u}} \in H_{0}^{1}\left(\Omega_{o}\right)^{d}=\left\{\boldsymbol{v} \in H^{1}\left(\Omega_{o}\right)^{d}:\left.\boldsymbol{v}\right|_{\Gamma_{\mathrm{D}}}=\mathbf{0}\right\}$ for which the body is in mechanical equilibrium. The resulting Euler-Lagrange equations associated to (1) read as: Find $\hat{\boldsymbol{u}} \in H_{0}^{1}\left(\Omega_{o}\right)^{d}$ and $\hat{p} \in L^{2}\left(\Omega_{o}\right)$ such that

$$
\begin{aligned}
-\operatorname{div} \mathbf{P}(\hat{\boldsymbol{u}}, \hat{p})=\mathbf{0} & \text { in } \Omega_{o}, \\
\hat{J}=1 & \text { in } \Omega_{o},
\end{aligned}
$$

in the sense of distributions, with the boundary data $\hat{\boldsymbol{u}}=\boldsymbol{g}$ on $\Gamma_{\mathrm{D}}$ (which will not be enforced in the choice of the functional space, but rather through a boundary flux term) and $\mathbf{P} \boldsymbol{n}=\boldsymbol{t}$ on $\Gamma_{\mathrm{N}}$, where $\hat{J}=\operatorname{det} \hat{\mathbf{F}}$ and $\mathbf{P}$ is the first PiolaKirchhoff stress tensor for incompressible materials

$$
\mathbf{P}=\frac{\partial \mathcal{W}}{\partial \hat{\mathbf{F}}}-\hat{p} \hat{J} \hat{\mathbf{F}}^{-T} .
$$

The variational formulation of problem (2) consists in finding $\hat{\boldsymbol{u}} \in H_{0}^{1}\left(\Omega_{o}\right)^{d}$ and $\hat{p} \in L^{2}\left(\Omega_{o}\right)$ such that

$$
\begin{aligned}
& A(\hat{\boldsymbol{u}}, \boldsymbol{v})=\int_{\Gamma_{\mathrm{N}}} \boldsymbol{t} \cdot \boldsymbol{v} d s \quad \text { for all } \boldsymbol{v} \in H_{0}^{1}\left(\Omega_{o}\right)^{d}, \\
& B(q, \hat{\boldsymbol{u}})=0 \quad \text { for all } q \in L^{2}\left(\Omega_{o}\right) .
\end{aligned}
$$

The nonlinear terms $A(\cdot, \cdot)$ and $B(\cdot, \cdot)$ are given by

$$
A(\hat{\boldsymbol{u}}, \boldsymbol{v}):=\int_{\Omega_{0}} \mathbf{P}(\hat{\boldsymbol{u}}, \hat{p}): \nabla \boldsymbol{v} d \boldsymbol{x}, \quad B(q, \hat{\boldsymbol{u}}):=\int_{\Omega_{0}} q(\hat{J}-1) d \boldsymbol{x} .
$$

The existence of solutions for (3) can be established following e.g. [17].

Our interest lies in the study of a special class of problems arising in the modeling of active deformations in soft biological tissues [14, 23], which under some assumptions, can be considered as hyperelastic materials. Here we restrict ourselves to a somewhat simplified setting, where one assumes that the passive behavior of the tissue can be described by a neo-Hookean constitutive law, whereas the active contribution is encoded in some additional anisotropic terms appearing after applying a so-called active strain decomposition [19]. In turn, the stress tensor is written as

$$
\mathbf{P}=\hat{\mathbf{F}} \Theta\left(\gamma_{\mathbf{f}_{o}}, \boldsymbol{x}\right)-\hat{p} \hat{J} \hat{\mathbf{F}}^{-T}
$$

with $\Theta\left(\gamma_{\mathbf{f}_{o}}, \boldsymbol{x}\right)=\mu \operatorname{det}\left(\mathbf{F}_{A}\right) \mathbf{F}_{A}^{-1} \mathbf{F}_{A}^{-T}$ and

$$
\mathbf{F}_{A}=\mathbf{I}+\gamma_{\mathbf{f}_{o}} \mathbf{f}_{o}(\boldsymbol{x}) \otimes \mathbf{f}_{o}(\boldsymbol{x})+\left[\left(1+\gamma_{\mathbf{f}_{o}}\right)^{-1 / 2}-1\right]\left(\mathbf{s}_{o}(\boldsymbol{x}) \otimes \mathbf{s}_{o}(\boldsymbol{x})+\mathbf{n}_{o}(\boldsymbol{x}) \otimes \mathbf{n}_{o}(\boldsymbol{x})\right) .
$$


Here $\mu$ is the neo-Hookean elastic modulus, $\gamma_{\mathbf{f}_{o}}$ is a smooth scalar activation function depending on ionic quantities and electrical signaling, and $\mathbf{f}_{o}(\boldsymbol{x}), \mathbf{s}_{o}(\boldsymbol{x}), \mathbf{n}_{o}(\boldsymbol{x})$ is a coordinate system pointing in the local direction of cardiac fibers, transversal sheet compound, and normal direction.

Using the relations

$$
D \hat{\mathbf{F}}^{-T}(\boldsymbol{u})=-\hat{\mathbf{F}}^{-T}(\nabla \boldsymbol{u})^{T} \hat{\mathbf{F}}^{-T}, \quad D \hat{J}(\boldsymbol{u})=\hat{J} \hat{\mathbf{F}}^{-T}: \nabla \boldsymbol{u}, \text { for all } \boldsymbol{u},
$$

we arrive at the following saddle-point problem coming from the linearization of (3) around the generic point $(\hat{\boldsymbol{u}}, \hat{p})$. Find infinitesimal increments $\boldsymbol{u} \in$ $H_{0}^{1}\left(\Omega_{o}\right)^{d}$ and $p \in L^{2}\left(\Omega_{o}\right)$ such that:

$$
\begin{aligned}
& a(\boldsymbol{u}, \boldsymbol{v})+b(p, \boldsymbol{v})=F(\boldsymbol{v}) \quad \text { for all } \boldsymbol{v} \in H_{0}^{1}\left(\Omega_{o}\right)^{d}, \\
& b(q, \boldsymbol{u})=G(q) \text { for all } q \in L^{2}\left(\Omega_{o}\right),
\end{aligned}
$$

where the involved bilinear and linear forms are defined as

$$
\begin{aligned}
a(\boldsymbol{u}, \boldsymbol{v}) & :=\int_{\Omega_{o}} \nabla \boldsymbol{u} \Theta\left(\gamma_{\mathbf{f}_{o}}, \boldsymbol{x}\right): \nabla \boldsymbol{v}+\hat{p} \hat{J}\left(\hat{\mathbf{F}}^{-1} \nabla \boldsymbol{u}\right)^{T}:\left(\hat{\mathbf{F}}^{-1} \nabla \boldsymbol{v}\right) d \boldsymbol{x}, \\
G(q) & :=-B(q, \hat{\boldsymbol{u}}), \quad b(p, \boldsymbol{v}):=-\int_{\Omega_{o}} p \hat{J} \hat{\mathbf{F}}^{-T}: \nabla \boldsymbol{v} d \boldsymbol{x} \\
F(\boldsymbol{v}) & :=A(\hat{\boldsymbol{u}}, \boldsymbol{v})-\int_{\Gamma_{\mathrm{N}}} \boldsymbol{t} \cdot \boldsymbol{v} d s-\int_{\Gamma_{\mathrm{D}}} \boldsymbol{g} \cdot \boldsymbol{v} d s
\end{aligned}
$$

In addition, we define the auxiliary quadrilinear form

$$
\mathcal{A}((\boldsymbol{u}, p),(\boldsymbol{v}, q)):=a(\boldsymbol{u}, \boldsymbol{v})+b(p, \boldsymbol{v})-b(q, \boldsymbol{u}) .
$$

We recall the usual notation $\|\cdot\|_{0, \Omega_{o}},\|\cdot\|_{1, \Omega_{o}}$ for $L^{2}-$ and $H^{1}$-norms, respectively.

The following theorem states the well-posedness of (5) (for a proof, see the general result for saddle-point problems in [11]). Of course, enough regularity should be assumed for the state $(\hat{\boldsymbol{u}}, \hat{p})$ so that the quantities in e.g. (4) and (5) are well defined.

Theorem 1 Problem (5) admits a unique solution $(\boldsymbol{u}, p) \in H_{0}^{1}\left(\Omega_{o}\right)^{d} \times L^{2}\left(\Omega_{o}\right)$. Moreover, there exists a constant $C>0$, not depending on the solution, such that

$$
\|\boldsymbol{u}\|_{1, \Omega_{o}}+\|p\|_{0, \Omega_{o}} \leq C\left(\|F\|_{\left(H_{0}^{1}\left(\Omega_{o}\right)^{d}\right)^{\prime}}+\|G\|_{H^{-1}\left(\Omega_{o}\right)}\right) .
$$

Notice that (5) corresponds to the linearization of (3) and therefore it can be regarded as a generic Newton (or Newton-like) step for the overall solution of (3). 


\section{A discontinuous Galerkin formulation}

\subsection{Admissible meshes and discrete gradient}

We consider a partition $\mathcal{T}_{h}$ of $\Omega_{o}$ consisting of geometrically conforming open simplicial elements $K$ (tetrahedra for $d=3$, or triangles for $d=2$ ), such that $\bar{\Omega}_{o}=\cup_{K \in \mathcal{T}_{h}}$. The mesh parameter is defined as $h=\max _{K \in \mathcal{T}_{h}} \operatorname{diam}(K)$. By $e \subset$ $\partial K$ we denote a face of a generic element $K \in \mathcal{T}_{h}$, of measure $h_{e}=|e|$. We assume that $\mathcal{T}_{h}$ is quasi-uniform, i.e., there exists $\rho>0$ such that

$$
\rho h<\inf _{K \in \mathcal{T}_{h}} \operatorname{diam}\left(B_{K}\right),
$$

where $B_{K}$ is the largest ball included in $K$. By $\mathcal{E}_{h}$ we denote the set of all element faces of $\mathcal{T}_{h}$, and $\mathcal{E}_{h}^{\text {int }} \subset \mathcal{E}_{h}$ denotes its restriction to internal faces. We will use the finite element spaces of polynomials of order $k$ and $k-1, k \geq 1$ (not necessarily continuous) defined on the mesh $\mathcal{T}_{h}$ :

$$
\begin{aligned}
\mathcal{V}_{h} & =\left\{\boldsymbol{v}_{h} \in L^{2}\left(\Omega_{o}\right)^{d}:\left.\boldsymbol{v}_{h}\right|_{K} \in \mathbb{P}^{k}(K)^{d}, \forall K \in \mathcal{T}_{h}\right\}, \\
Q_{h} & =\left\{q_{h} \in L^{2}\left(\Omega_{o}\right)^{d}:\left.q_{h}\right|_{K} \in \mathbb{P}^{k-1}(K), \forall K \in \mathcal{T}_{h}\right\} .
\end{aligned}
$$

Since functions in the aforementioned spaces are allowed to be discontinuous across element boundaries, we recall the standard notation for jump and average operators over the face $e$ shared by the two neighboring elements $K$ and $L$ :

$$
\begin{aligned}
& \llbracket \boldsymbol{v}_{h} \rrbracket=\left.\boldsymbol{v}_{h}\right|_{K}-\left.\boldsymbol{v}_{h}\right|_{L}, \llbracket q_{h} \rrbracket=\left.q_{h}\right|_{K}-\left.q_{h}\right|_{L}, \\
& \left\{\left\{\boldsymbol{v}_{h}\right\}\right\}=\frac{1}{2}\left(\left.\boldsymbol{v}_{h}\right|_{K}+\left.\boldsymbol{v}_{h}\right|_{L}\right),\left\{\left\{\mathbf{W}_{h}\right\}\right\}=\frac{1}{2}\left(\left.\mathbf{W}_{h}\right|_{K}+\left.\mathbf{W}_{h}\right|_{L}\right),
\end{aligned}
$$

for $\boldsymbol{v}_{h} \in \mathcal{V}_{h}, q_{h} \in Q_{h}, \mathbf{W}_{h} \in \mathcal{V}_{h}^{d}$.

We will also make use of the DG gradient defined as

$$
\nabla_{\mathrm{DG}} \boldsymbol{v}_{h}=\nabla \boldsymbol{v}_{h}+\mathcal{R} \llbracket \boldsymbol{v}_{h} \rrbracket, \quad \nabla_{\mathrm{DG}} q_{h}=\nabla q_{h}+\mathcal{R} \llbracket q_{h} \rrbracket,
$$

for all $\boldsymbol{v}_{h} \in \mathcal{V}_{h}, q_{h} \in Q_{h}$, where $\mathcal{R}: L^{2}\left(\mathcal{E}_{h}^{\text {int }}\right) \rightarrow Q_{h}^{d}$ and $\mathcal{R}: L^{2}\left(\mathcal{E}_{h}^{\text {int }}\right)^{d} \rightarrow \mathcal{V}_{h}^{d}$ are lifting operators (see [25]) defined by the relations

$$
\begin{aligned}
& \int_{\Omega_{o}} \mathcal{R}\left(q_{h}\right) \cdot \boldsymbol{w}_{h} d \boldsymbol{x}=\int_{\mathcal{E}_{h}^{\text {int }}} q_{h}\left\{\left\{\boldsymbol{w}_{h}\right\}\right] \cdot \boldsymbol{n} d s \quad \forall \boldsymbol{w}_{h} \in Q_{h}^{d}, \\
& \int_{\Omega_{o}} \mathcal{R}\left(\boldsymbol{v}_{h}\right): \mathbf{W}_{h} d \boldsymbol{x}=\int_{\mathcal{E}_{h}^{\text {int }}} \boldsymbol{v}_{h} \otimes \boldsymbol{n}:\left\{\left\{\mathbf{W}_{h}\right\}\right\} d s \quad \forall \mathbf{W}_{h} \in \mathcal{V}_{h}^{d} .
\end{aligned}
$$

These operators satisfy the relation

$$
C_{1}\left\|\mathcal{R} \llbracket \boldsymbol{v}_{h} \rrbracket\right\|_{0, \Omega_{o}} \leq h^{-1 / 2}\left\|\llbracket \boldsymbol{v}_{h} \rrbracket\right\|_{0, \mathcal{E}_{h}^{\text {int }}} \leq C_{2}\left\|\mathcal{R} \llbracket \boldsymbol{v}_{h} \rrbracket\right\|_{0, \Omega_{o}},
$$

for all $\boldsymbol{v}_{h} \in \mathcal{V}_{h}^{d}$, and the obvious analogous for $\mathcal{R}$ (see e.g. [18]). Here $C_{1}, C_{2}$ are positive constants independent of $h$. Similar inequalities hold for their restrictions to the generic face $e \in \mathcal{E}_{h}^{\text {int }}:\left.\mathcal{R}\right|_{e}: L^{2}(e) \rightarrow Q_{h}^{d}$ and $\left.\mathcal{R}\right|_{e}: L^{2}(e)^{d} \rightarrow$ $\mathcal{V}_{h}^{d}$ 
Lemma 1 The following estimate holds for all $\boldsymbol{w}_{h}, \boldsymbol{v}_{h} \in H^{k}(K)^{d}$, for $e \subset \partial K$, $K \in \mathcal{T}_{h}$

$$
\int_{e}\left\{\left\{\nabla \boldsymbol{w}_{h} \boldsymbol{n}\right\}\right\} \cdot \llbracket \boldsymbol{v}_{h} \rrbracket d s \leq C \sum_{e \subset \partial K} h_{e}^{-1 / 2}\left|\boldsymbol{w}_{h}\right|_{1, K}\left\|\llbracket \llbracket \boldsymbol{v}_{h} \rrbracket\right\| \|_{0, e} .
$$

For a proof see [16]. The following trace inequality can be found in e.g. [9].

Lemma 2 Let $\boldsymbol{v}_{h} \in H^{k}(K)^{d}$, and $e \subset \partial K$, for a generic element $K \in \mathcal{T}_{h}$. Then we have

$$
\left\|\boldsymbol{v}_{h}\right\|_{0, e}^{2} \leq C\left(h_{K}^{-1}\left\|\boldsymbol{v}_{h}\right\|_{0, K}^{2}+h_{K}\left|\boldsymbol{v}_{h}\right|_{1, K}^{2}\right), \quad\left\|\partial_{\boldsymbol{n}} \boldsymbol{v}_{h}\right\|_{0, e}^{2} \leq C\left(h_{e}^{-1}\left|\boldsymbol{v}_{h}\right|_{1, K}^{2}+h_{e}\left|\boldsymbol{v}_{h}\right|_{2, K}^{2}\right) .
$$

\subsection{Stabilized interior penalty DG formulation}

The stabilized DG approximation of (5) that we will analyze corresponds to finding $\left(\boldsymbol{u}_{h}, p_{h}\right) \in \mathcal{V}_{h} \times Q_{h}$ such that:

$$
\begin{aligned}
& a_{h}\left(\boldsymbol{u}_{h}, \boldsymbol{v}_{h}\right)+c_{h}\left(\boldsymbol{u}_{h}, \boldsymbol{v}_{h}\right)+b_{h}\left(p_{h}, \boldsymbol{v}_{h}\right)=F\left(\boldsymbol{v}_{h}\right) \text { for all } \boldsymbol{v}_{h} \in \mathcal{V}_{h}, \\
& b_{h}\left(q_{h}, \boldsymbol{u}_{h}\right)+d_{h}\left(p_{h}, q_{h}\right)=G\left(q_{h}\right) \quad \text { for all } q_{h} \in Q_{h},
\end{aligned}
$$

where the discrete counterparts $a_{h}(\cdot, \cdot), b_{h}(\cdot, \cdot)$ of the bilinear forms $a(\cdot, \cdot)$, $b(\cdot, \cdot)$ are given by

$$
\begin{aligned}
a_{h}\left(\boldsymbol{u}_{h}, \boldsymbol{v}_{h}\right):= & \sum_{K \in \mathcal{T}_{h}} \int_{K} \nabla \boldsymbol{u}_{h} \Theta\left(\gamma_{\mathbf{f}_{o}}, \boldsymbol{x}\right): \nabla \boldsymbol{v}_{h}+\hat{p}_{h} \hat{J}\left(\hat{\mathbf{F}}^{-1} \nabla \boldsymbol{u}_{h}\right)^{T}:\left(\hat{\mathbf{F}}^{-1} \nabla \boldsymbol{v}_{h}\right) d \boldsymbol{x} \\
& +\sum_{e \in \mathcal{E}_{h}^{\text {int }}} \int_{e}\left\{\left\{\nabla \boldsymbol{u}_{h} \Theta\left(\gamma_{\mathbf{f}_{o}}, \boldsymbol{x}\right) \boldsymbol{n}\right\}\right\} \cdot \llbracket \boldsymbol{v}_{h} \rrbracket d s \\
& +\sum_{e \in \mathcal{E}_{h}^{\text {int }}} \int_{e}\left\{\left\{\hat{p}_{h} \hat{J}\left(\hat{\mathbf{F}}^{-1} \nabla \boldsymbol{u}_{h}\right)^{T} \boldsymbol{n}\right\}\right\} \cdot \llbracket \hat{\mathbf{F}}^{-1} \boldsymbol{v}_{h} \rrbracket d s, \\
b_{h}\left(p_{h}, \boldsymbol{v}_{h}\right):= & -\sum_{K \in \mathcal{T}_{h}} \int_{K} p_{h} \hat{J} \hat{\mathbf{F}}^{-T}: \nabla \boldsymbol{v}_{h} d \boldsymbol{x}-\sum_{e \in \mathcal{E}_{h}^{\text {int }}} \int_{e}\left\{\left\{p_{h} \hat{J} \hat{\mathbf{F}}^{-T} \boldsymbol{n}\right\}\right\} \cdot \llbracket \boldsymbol{v}_{h} \rrbracket d s .
\end{aligned}
$$

Moreover, we have set

$$
\begin{aligned}
c_{h}\left(\boldsymbol{u}_{h}, \boldsymbol{v}_{h}\right) & :=\beta \sum_{e \in \mathcal{E}_{h}^{\text {int }}} \int_{e} \frac{1}{h_{e}} \llbracket \boldsymbol{u}_{h} \rrbracket \cdot \llbracket \boldsymbol{v}_{h} \rrbracket d s+\beta \sum_{e \in \mathcal{E}_{h} \cap \Gamma_{\mathrm{D}}} \int_{e} \frac{1}{h_{e}} \boldsymbol{u}_{h} \cdot \boldsymbol{v}_{h} d s, \\
d_{h}\left(p_{h}, q_{h}\right): & =\delta \sum_{K \in \mathcal{T}_{h}} \int_{K} p_{h} q_{h} \hat{\mathbf{F}}: \mathbf{I} d \boldsymbol{x}+\alpha \sum_{e \in \mathcal{E}_{h}^{\text {int }}} \int_{e} h_{e} \llbracket p_{h} \rrbracket \llbracket q_{h} \rrbracket d s .
\end{aligned}
$$

Here $\alpha, \beta, \delta$ are stabilization parameters. Notice that by definition of $F_{h}$, the integral on the edges lying on $\Gamma_{\mathrm{D}}$ corresponds to the weak imposition of $\boldsymbol{u}_{h}=\boldsymbol{g}$ (see (7a)) and the second term in the LHS penalizes jump and 
normal jump of the deformation field. As usual, the choice of $\alpha, \beta, \delta$ is dictated by stability requirements. More precisely, the penalty parameter $\beta$ needs to assume sufficiently large values so that the coercivity of $a_{h}(\cdot, \cdot)+c_{h}(\cdot, \cdot)$ is ensured. Next, defining the forms

$$
\begin{aligned}
\mathcal{A}_{h}\left(\left(\boldsymbol{u}_{h}, p_{h}\right),\left(\boldsymbol{v}_{h}, q_{h}\right)\right):= & a_{h}\left(\boldsymbol{u}_{h}, \boldsymbol{v}_{h}\right)+b_{h}\left(p_{h}, \boldsymbol{v}_{h}\right)-b_{h}\left(q_{h}, \boldsymbol{u}_{h}\right) \\
& +c_{h}\left(\boldsymbol{u}_{h}, \boldsymbol{v}_{h}\right)-d_{h}\left(p_{h}, q_{h}\right), \\
\mathcal{F}_{h}\left(\boldsymbol{v}_{h}, q_{h}\right):= & F\left(\boldsymbol{v}_{h}\right)-G\left(q_{h}\right),
\end{aligned}
$$

system (7) reads: Find $\left(\boldsymbol{u}_{h}, p_{h}\right) \in \mathcal{V}_{h} \times Q_{h}$ such that

$$
\mathcal{A}_{h}\left(\left(\boldsymbol{u}_{h}, p_{h}\right),\left(\boldsymbol{v}_{h}, q_{h}\right)\right)=\mathcal{F}_{h}\left(\boldsymbol{v}_{h}, q_{h}\right), \quad \text { for all }\left(\boldsymbol{v}_{h}, q_{h}\right) \in \mathcal{V}_{h} \times Q_{h} .
$$

We will perform a convergence analysis for (7a) and (7b) using the following energy norms

$\left\|\boldsymbol{v}_{h}\right\|_{h}^{2}:=\sum_{K \in \mathcal{T}_{h}}\left|\boldsymbol{v}_{h}\right|_{1, K}^{2}+\sum_{e \in \mathcal{E}_{h}^{\text {int }}} \frac{1}{h_{e}}\left\|\llbracket \boldsymbol{v}_{h} \rrbracket\right\|_{0, e}^{2}, \quad\left\|q_{h}\right\|_{h}^{2}:=\left\|q_{h}\right\|_{0, \Omega_{o}}^{2}+\sum_{e \in \mathcal{E}_{h}^{\text {int }}} h_{e}\left\|\llbracket q_{h} \rrbracket\right\|_{0, e}^{2}$.

By $\mathbf{I}_{h}: C^{0}\left(\Omega_{o}\right) \rightarrow \mathcal{V}_{h}$ and $\Xi: L^{2}\left(\Omega_{o}\right) \rightarrow V_{h}^{k}$ we will denote a discontinuous polynomial interpolation (such as e.g. the Brezzi-Douglas-Marini interpolant [6]) and the Clément operator, respectively [9]. We collect some useful interpolation estimates (see [22]) in the next lemma.

Lemma 3 Let $v \in H^{s}\left(\Omega_{o}\right)^{d}$ and $q \in H^{s-1}\left(\Omega_{o}\right)$, for $1 \leq s \leq k+1$. Then, the following inequalities hold

$$
\begin{aligned}
\left|\boldsymbol{v}-\mathbf{I}_{h} \boldsymbol{v}\right|_{m, K} & \leq C h^{s-m}|\boldsymbol{v}|_{s, K}, \\
\left|q-\boldsymbol{\Xi}_{h} q\right|_{m, K} & \leq C h^{s-1-m}|q|_{s-1, K}, \\
\left\|\boldsymbol{v}-\mathbf{I}_{h} \boldsymbol{v}\right\|_{h} & \leq C h^{s-1}|\boldsymbol{v}|_{s, \Omega_{o}}, \\
\left\|\mathbf{I}_{h} \boldsymbol{v}\right\|_{h} & \leq C|\boldsymbol{v}|_{s, \Omega_{o}}, \\
\left\|q-\Xi_{h} q\right\|_{h} & \leq C h^{s-1}|q|_{s-1, \Omega_{o}},
\end{aligned}
$$

where $0 \leq m \leq 1$ and $C>0$.

Proof Estimates (9), (10) are deduced in e.g. [1, 9]. With these, triangular inequality and Lemma 2 we get the estimate (11) as follows

$$
\begin{aligned}
\left\|\boldsymbol{v}-\mathbf{I}_{h} \boldsymbol{v}\right\|_{h}^{2} & \leq\left|\boldsymbol{v}-\mathbf{I}_{h} \boldsymbol{v}\right|_{1, \Omega_{o}}^{2}+\sum_{e \in \mathcal{E}_{h}^{\mathrm{int}}} h_{e}^{-1}\left\|\llbracket \boldsymbol{v}-\mathbf{I}_{h} \boldsymbol{v} \rrbracket\right\|_{0, e}^{2}+\sum_{K \in \mathcal{T}_{h}} h_{K}^{2}\left|\boldsymbol{v}-\mathbf{I}_{h} \boldsymbol{v}\right|_{2, K}^{2} \\
& \leq C h^{2(s-1)}|\boldsymbol{v}|_{S, \Omega_{o}}^{2}+C \sum_{K \in \mathcal{T}_{h}}\left(h_{K}^{-2}\left\|\boldsymbol{v}-\mathbf{I}_{h} \boldsymbol{v}\right\|_{0, K}^{2}+h_{K}^{2}\left|\boldsymbol{v}-\mathbf{I}_{h} \boldsymbol{v}\right|_{2, K}^{2}\right) \\
& \leq C h^{2(s-1)}|\boldsymbol{v}|_{s, \Omega_{o}}^{2},
\end{aligned}
$$

and an analogous argument leads to (12) and (13). 
Proposition 1 The quadrilinear form $\mathcal{A}_{h}(\cdot, \cdot)$ is continuous and coercive in the energy norm.

Proof The continuity of $\mathcal{A}_{h}$ is a direct result of Cauchy-Schwarz inequality, definition of mesh-dependent norms, and Lemma 1 with $\boldsymbol{w}_{h}=\hat{\boldsymbol{u}}$. For the coercivity, definition of $\mathcal{A}_{h}$, triangular inequality, tensor Young's inequality, boundedness of the lifting operators (6), mesh regularity, and standard inverse inequality give

$$
\begin{aligned}
\left|\mathcal{A}_{h}\left(\left(\boldsymbol{u}_{h}, p_{h}\right),\left(\boldsymbol{u}_{h}, p_{h}\right)\right)\right| \geq & \left|a_{h}\left(\boldsymbol{u}_{h}, \boldsymbol{u}_{h}\right)+c_{h}\left(\boldsymbol{u}_{h}, \boldsymbol{u}_{h}\right)\right|+\left|b_{h}\left(p_{h}, \boldsymbol{u}_{h}\right)\right| \\
& -\left|b_{h}\left(p_{h}, \boldsymbol{u}_{h}\right)\right|-\left|d_{h}\left(p_{h}, p_{h}\right)\right| \\
= & \left|a_{h}\left(\boldsymbol{u}_{h}, \boldsymbol{u}_{h}\right)+c_{h}\left(\boldsymbol{u}_{h}, \boldsymbol{u}_{h}\right)\right|-\left|d_{h}\left(p_{h}, p_{h}\right)\right| \\
\geq & C \min \left\{(1-\varepsilon),\left(\|\Theta\|_{0, \Omega_{o}}+\beta\right)-\|\{\Theta\}\|_{\infty, \Omega_{o}} \varepsilon^{-1}\right\}\left\|\boldsymbol{u}_{h}\right\|_{h}^{2} \\
& +\alpha h_{e}^{-1}\left\|p_{h}\right\|_{h}^{2} \\
\geq & C\left(\left\|\boldsymbol{u}_{h}\right\|_{h}^{2}+\left\|p_{h}\right\|_{h}^{2}\right), \quad \forall\left(\boldsymbol{u}_{h}, p_{h}\right) \in \mathcal{V}_{h} \times Q_{h} .
\end{aligned}
$$

Notice that the continuity and coercivity constants may depend on the fixed state around which the linearization is performed, and the activation term $\Theta$, but they do not depend on the meshsize, nor on the solution itself. ${ }^{1}$ This is due to the fact that the linearization is done only once on the continuous nonlinear problem. However, the nonlinear nature of (3) implies that large loads can cause a breakdown of the mechanical system [5], and therefore the result is valid within a so-called region of stability.

Next, we assess the consistency of the method.

Proposition 2 Let $(\boldsymbol{u}, p)$ be the unique solution of (5). Then, the discontinuous Galerkin formulation (7a) and (7b) is asymptotically consistent, that is

$$
\mathcal{A}_{h}\left(\left(\boldsymbol{u}-\boldsymbol{u}_{h}, p-p_{h}\right),\left(\boldsymbol{v}_{h}, q_{h}\right)\right) \leq C h\left\|\boldsymbol{v}_{h}\right\|_{h},
$$

where $C=C(\hat{\boldsymbol{u}}, \hat{p}, \Theta, F, G)>0$. In particular, if $\mathcal{V}_{h} \times Q_{h}$ is a subspace of the the corresponding space of piecewise continuous functions, then the usual strong consistency relation is recovered

$$
\mathcal{A}_{h}\left(\left(\boldsymbol{u}-\boldsymbol{u}_{h}, p-p_{h}\right),\left(\boldsymbol{v}_{h}, q_{h}\right)\right)=0 .
$$

\footnotetext{
${ }^{1}$ Indeed, the continuity and coercivity constants are explicitly given by

$$
\begin{aligned}
C_{\text {cont }} & =\max \left\{C_{\Omega_{o}}\left(\|\Theta\|_{\infty, \Omega_{o}}+C_{0}(|\nabla \hat{u}|)\|\hat{p}\|_{0}\right),\|\Theta\|_{\infty, \Omega_{o}}^{2},\left\|\hat{J} \hat{p}\left(\nabla \hat{\boldsymbol{u}}^{T}\right)^{2}\right\|_{0, \Omega_{o}}, \beta, C_{1}(\hat{\boldsymbol{u}}), \alpha\right\}, \\
C_{\text {coerc }} & =C_{2}(\hat{\boldsymbol{u}}, \hat{p}) \min \left\{\|\Theta\|_{0, \Omega_{o}}+\beta-\|\{\Theta\}\|_{\infty, \Omega_{o}} \varepsilon^{-1}, C_{3} \alpha\right\},
\end{aligned}
$$
}

where $C_{\Omega_{o}}$ is a constant depending on the shape of the domain. 
Proof The assumed regularity for the exact solution implies that $\llbracket \boldsymbol{u} \rrbracket=\mathbf{0}$, $\llbracket p \rrbracket=0$. In addition, (8) followed by definition of continuous and discrete bilinear forms, Hölder inequality, Theorem 1, and definition of mesh-dependent norm, yields

$$
\begin{aligned}
\mathcal{A}_{h}((\boldsymbol{u} & \left.\left.-\boldsymbol{u}_{h}, p-p_{h}\right),\left(\boldsymbol{v}_{h}, q_{h}\right)\right) \\
= & a_{h}\left(\boldsymbol{u}, \boldsymbol{v}_{h}\right)+b_{h}\left(p, \boldsymbol{v}_{h}\right)-b_{h}\left(q_{h}, \boldsymbol{u}\right)-d_{h}\left(p, q_{h}\right)-\mathcal{F}_{h}\left(\boldsymbol{v}_{h}, q_{h}\right) \\
= & a\left(\boldsymbol{u}, \boldsymbol{v}_{h}\right)+\sum_{e \in \mathcal{E}_{h}^{\text {int }}} \int_{e} \nabla \boldsymbol{u} \Theta\left(\gamma_{\mathbf{f}_{o}}, \boldsymbol{x}\right) \boldsymbol{n} \cdot \llbracket \boldsymbol{v}_{h} \rrbracket d s \\
& +\sum_{e \in \mathcal{E}_{h}^{\text {int }}} \int_{e} \hat{p} \hat{J}\left(\hat{\mathbf{F}}^{-1} \nabla \boldsymbol{u}\right)^{T} \boldsymbol{n} \cdot \hat{\mathbf{F}}^{-1} \llbracket \boldsymbol{v}_{h} \rrbracket d s \\
& +b\left(p, \boldsymbol{v}_{h}\right)-\sum_{e \in \mathcal{E}_{h}^{\text {int }}} \int_{e} p \hat{J} \hat{\mathbf{F}}^{-T} \boldsymbol{n} \cdot \llbracket \boldsymbol{v}_{h} \rrbracket d s-b\left(q_{h}, \boldsymbol{u}\right)-\mathcal{F}_{h}\left(\boldsymbol{v}_{h}, q_{h}\right) \\
= & \sum_{e \in \mathcal{E}_{h}^{\text {int }}} \int_{e} \nabla \boldsymbol{u} \Theta\left(\gamma_{\mathbf{f}_{o}}, \boldsymbol{x}\right) \boldsymbol{n} \cdot \llbracket \boldsymbol{v}_{h} \rrbracket d s+\sum_{e \in \mathcal{E}_{h}^{\text {int }}} \int_{e} \hat{p} \hat{J}\left(\hat{\mathbf{F}}^{-1} \nabla \boldsymbol{u}\right)^{T} \boldsymbol{n} \cdot \hat{\mathbf{F}}^{-1} \llbracket \boldsymbol{v}_{h} \rrbracket d s \\
& -\sum_{e \in \mathcal{E}_{h}^{\text {int }}} \int_{e} p \hat{J} \hat{\mathbf{F}}^{-T} \boldsymbol{n} \cdot \llbracket \boldsymbol{v}_{h} \rrbracket d s \\
\leq & C(\Theta, \hat{\boldsymbol{u}}, \hat{p})\left(\sum_{e \in \mathcal{E}_{h}^{\text {int }}}\|\nabla \boldsymbol{u}\|_{0, e}^{2}\left\|\llbracket \boldsymbol{v}_{h} \rrbracket\right\|_{0, e}^{2}\right)^{1 / 2}+C(\hat{\boldsymbol{u}})\left(\sum_{e \in \mathcal{E}_{h}^{\text {int }}}\|p\|_{0, e}^{2}\left\|\llbracket \boldsymbol{v}_{h} \rrbracket\right\|_{0, e}^{2}\right)^{1 / 2} \\
\leq & C(\Theta, \hat{\boldsymbol{u}}, \hat{p})\left(\|F\|_{\left(H_{0}^{1}\left(\Omega_{o}\right)\right)^{\prime}}^{2}+\|G\|_{H^{-1}\left(\Omega_{o}\right)}^{2}\right)^{1 / 2}\left(\sum_{e \in \mathcal{E}_{h}^{\text {int }}}\left\|\llbracket \boldsymbol{v}_{h} \rrbracket\right\|_{0, e}^{2}\right)^{1 / 2} \\
\leq & C(\Theta, \hat{\boldsymbol{u}}, \hat{p}, F, G) h\left\|\boldsymbol{v}_{h}\right\|_{h} .
\end{aligned}
$$

Finally, assertion (14) follows directly from (15).

\subsection{Convergence analysis}

Let us now turn to the derivation of error estimates for (7a) and (7b). Notice that this corresponds to a linear analysis, and therefore elements that satisfy the discrete inf-sup condition may exhibit instabilities when applied to large deformation elasticity [2]. However, as we will see in the experiments of Section 4, no instabilities are observed for our discontinuous formulation.

Theorem 2 Let us assume that $\left(\boldsymbol{u}_{h}, p_{h}\right) \in \mathcal{V}_{h} \times Q_{h}$ is the unique solution of (7a) and $(7 \mathrm{~b})$ and $(\boldsymbol{u}, p) \in H^{k+1}\left(\Omega_{o}\right)^{d} \times H^{k}\left(\Omega_{o}\right)$ that of (5). Then, there exists $C>0$ such that

$$
\left\|\boldsymbol{u}-\boldsymbol{u}_{h}\right\|_{h}+\left\|p-p_{h}\right\|_{h} \leq C h^{k}\left(|\boldsymbol{u}|_{k+1, \Omega_{o}}+|p|_{k, \Omega_{o}}\right) .
$$


Proof Propositions 1 and 2, along with Cauchy-Schwarz inequality imply that

$$
\begin{aligned}
& \left\|\mathbf{I}_{h} \boldsymbol{u}-\boldsymbol{u}_{h}\right\|_{h}^{2}+\left\|\boldsymbol{\Xi}_{h} p-p_{h}\right\|_{h}^{2} \\
& \leq \mathcal{A}_{h}\left(\left(\mathbf{I}_{h} \boldsymbol{u}-\boldsymbol{u}_{h}, \Xi_{h} p-p_{h}\right),\left(\mathbf{I}_{h} \boldsymbol{u}-\boldsymbol{u}_{h}, \Xi_{h} p-p_{h}\right)\right) \\
& \leq \mathcal{A}_{h}\left(\left(\mathbf{I}_{h} \boldsymbol{u}-\boldsymbol{u}, \boldsymbol{\Xi}_{h} p-p\right),\left(\mathbf{I}_{h} \boldsymbol{u}-\boldsymbol{u}_{h}, \boldsymbol{\Xi}_{h} p-p_{h}\right)\right)+h\left\|\mathbf{I}_{h} \boldsymbol{u}-\boldsymbol{u}_{h}\right\|_{h} \\
& \leq C\left(\left|\mathbf{I}_{h} \boldsymbol{u}-\boldsymbol{u}\right|_{1, \Omega_{o}}^{2}+\sum_{e \in \mathcal{E}_{h}^{\text {int }}}\left|\left\{\mathbf{I}_{h} \boldsymbol{u}-\boldsymbol{u}\right\} \boldsymbol{n}\right|_{1, e}^{2}+\left\|\boldsymbol{\Xi}_{h} p-p\right\|_{0, \Omega_{o}}^{2}\right. \\
& +\sum_{e \in \mathcal{E}_{h}^{\text {int }}}\left\|\left\{\Xi_{h} p-p\right\}\right\|_{0, e}^{2}+\sum_{e \in \mathcal{E}_{h}^{\text {int }}} \alpha h_{e}\left|\llbracket \Xi_{h} p-p \rrbracket\right|_{1, e}^{2} \\
& +\sum_{e \in \mathcal{E}_{h}^{\mathrm{int}}} \beta h_{e}^{-1} \mid \llbracket\left[\mathbf{I}_{h} \boldsymbol{u}-\left.\boldsymbol{u} \rrbracket \boldsymbol{n}\right|_{1, e} ^{2}\right)^{1 / 2} \\
& \times\left(\left|\mathbf{I}_{h} \boldsymbol{u}-\boldsymbol{u}_{h}\right|_{1, \Omega_{o}}^{2}+\sum_{e \in \mathcal{E}_{h}^{\text {int }}}\left(1+\beta h_{e}^{-1}\right)\left\|\llbracket \mathbf{I}_{h} \boldsymbol{u}-\boldsymbol{u}_{h}\right\| \boldsymbol{n}\left\|_{0, e}^{2}+\right\| \Xi_{h} p-p_{h} \|_{0, \Omega_{o}}^{2}\right. \\
& \left.+\sum_{e \in \mathcal{E}_{h}^{\text {int }}}\left\|\left\{\Xi_{h} p-p_{h}\right\}\right\|_{0, e}^{2}+\sum_{e \in \mathcal{E}_{h}^{\text {int }}} \alpha h_{e}\left\|\llbracket \Xi_{h} p-p_{h} \rrbracket\right\|_{0, e}^{2}+h^{2}\left\|\mathbf{I}_{h} \boldsymbol{u}-\boldsymbol{u}_{h}\right\|_{h}^{2}\right)^{1 / 2} \\
& \leq C h^{k}\left(|\boldsymbol{u}|_{k+1, \Omega_{o}}^{2}+|p|_{k, \Omega_{o}}^{2}\right)^{1 / 2}\left(\left\|\mathbf{I}_{h} \boldsymbol{u}-\boldsymbol{u}_{h}\right\|_{h}^{2}+\left\|\Xi_{h} p-p_{h}\right\|_{h}^{2}\right)^{1 / 2},
\end{aligned}
$$

where the last relation follows from Lemmas 1 and 3. The proof is completed after applying triangular inequality and Lemma 3 once again.

Remark 1 Notice that if we only assume $p \in H^{k-1}\left(\Omega_{o}\right)$, then the convergence result from Theorem 2 reads

$$
\left\|\boldsymbol{u}-\boldsymbol{u}_{h}\right\|_{h}+\left\|p-p_{h}\right\|_{h} \leq C\left(h^{k}|\boldsymbol{u}|_{k+1, \Omega_{o}}+h^{k-1}|p|_{k, \Omega_{o}}\right) .
$$

Theorem 3 Under the same assumptions of Theorem 2, there exists $C>0$ such that

$$
\left\|\boldsymbol{u}-\boldsymbol{u}_{h}\right\|_{0, \Omega_{o}}+\left\|p-p_{h}\right\|_{0, \Omega_{o}} \leq C h^{k}\left(h^{k-1}|\boldsymbol{u}|_{k+1, \Omega_{o}}+|p|_{k, \Omega_{o}}\right) .
$$

Proof Our proof will be based on a classical duality argument. Let $(\boldsymbol{w}, \xi)$ be the unique solution of an auxiliary problem whose strong formulation is

$$
\begin{aligned}
-\operatorname{div}\left(\Theta \nabla \boldsymbol{w}+\hat{p} \hat{J} \hat{\mathbf{F}}^{-T} \nabla \boldsymbol{w} \hat{\mathbf{F}}^{-T}-\xi \hat{J} \hat{\mathbf{F}}^{-T}\right) & =\boldsymbol{u}-\boldsymbol{u}_{h}, \\
\operatorname{div}\left(\hat{J} \hat{\mathbf{F}}^{-T} \boldsymbol{w}\right) & =p-p_{h} .
\end{aligned}
$$

The existence of $(\boldsymbol{w}, \xi)$ is guaranteed by the continuous inf-sup condition satisfied by the spaces pair $H^{k}\left(\Omega_{o}\right)^{d} \times H^{k-1}\left(\Omega_{o}\right)$, and by the assumed 
regularity of the domain $\Omega_{o}$ (convex and bounded polygon or polyhedron) [11]. In addition, the following continuous dependence on the datum is satisfied

$$
|\boldsymbol{w}|_{k+1, \Omega_{o}}+|\xi|_{k, \Omega_{o}} \leq C\left\|\boldsymbol{u}-\boldsymbol{u}_{h}\right\|_{0, \Omega_{o}}, \quad|\boldsymbol{w}|_{k+1, \Omega_{o}} \leq C\left\|p-p_{h}\right\|_{0, \Omega_{o}} .
$$

Using the quadrilinear form $\mathcal{A}((\cdot, \cdot),(\cdot, \cdot))$, the weak form of (16) reads: Find $(\boldsymbol{w}, \xi)$ such that

$\mathcal{A}((\boldsymbol{w}, \xi),(\boldsymbol{v}, 0))=\int_{\Omega_{o}}\left(\boldsymbol{u}-\boldsymbol{u}_{h}\right) \cdot \boldsymbol{v} d \boldsymbol{x}, \quad \mathcal{A}((\boldsymbol{w}, 0),(0, q))=\int_{\Omega_{o}}\left(p-p_{h}\right) q d \boldsymbol{x}$,

for all $(\boldsymbol{v}, q)$, which holds in particular for $(\boldsymbol{v}, q)=\left(\boldsymbol{u}-\boldsymbol{u}_{h}, p-p_{h}\right)$. Combining this and Proposition 2, we readily obtain the inequalities

$$
\begin{aligned}
& \left|\mathcal{A}\left((\boldsymbol{w}, \xi),\left(\boldsymbol{u}-\boldsymbol{u}_{h}, 0\right)\right)-\left\|\boldsymbol{u}-\boldsymbol{u}_{h}\right\|_{0, \Omega_{o}}^{2}\right| \\
& \quad \leq C\left(h^{k}|\boldsymbol{w}|_{k+1, \Omega_{o}}+h^{k-1}|\xi|_{k, \Omega_{o}}\right)\left\|\boldsymbol{u}-\boldsymbol{u}_{h}\right\|_{h}, \\
& \quad\left|\mathcal{A}\left((\boldsymbol{w}, 0),\left(0, p-p_{h}\right)\right)-\left\|p-p_{h}\right\|_{0, \Omega_{o}}^{2}\right| \leq C h^{k}|\boldsymbol{w}|_{k+1, \Omega_{o}}\left\|p-p_{h}\right\|_{h} .
\end{aligned}
$$

Then we write $\boldsymbol{w}=\boldsymbol{w}-\mathbf{I}_{h} \boldsymbol{w}+\mathbf{I}_{h} \boldsymbol{w}, \xi=\xi-\boldsymbol{\Xi}_{h} \xi+\Xi_{h} \xi$, and after applying the continuity of the bilinear forms $\mathcal{A}$ and $\mathcal{A}_{h}$, together with Proposition 2, we arrive at

$$
\begin{aligned}
\left\|\boldsymbol{u}-\boldsymbol{u}_{h}\right\|_{0, \Omega_{o}}^{2} \leq & C\left(h^{k}|\boldsymbol{w}|_{k+1, \Omega_{o}}+h^{k-1}|\xi|_{k, \Omega_{o}}\right)\left\|\boldsymbol{u}-\boldsymbol{u}_{h}\right\|_{h} \\
& +C\left(\left\|\boldsymbol{w}-\mathbf{I}_{h} \boldsymbol{w}\right\|_{h}+\left\|\xi-\Xi_{h} \xi\right\|_{h}\right)\left\|\boldsymbol{u}-\boldsymbol{u}_{h}\right\|_{h}, \\
\left\|p-p_{h}\right\|_{0, \Omega_{o}}^{2} \leq C & h^{k}|\boldsymbol{w}|_{k+1, \Omega_{o}}\left\|p-p_{h}\right\|_{h} \\
& +C\left(\left\|\boldsymbol{w}-\mathbf{I}_{h} \boldsymbol{w}\right\|_{h}+\left\|\xi-\Xi_{h} \xi\right\|_{h}\right)\left\|p-p_{h}\right\|_{h} .
\end{aligned}
$$

Therefore, Lemma 3 and (17) yield

$$
\left\|\boldsymbol{u}-\boldsymbol{u}_{h}\right\|_{0, \Omega_{o}} \leq C h^{k-1}\left\|\boldsymbol{u}-\boldsymbol{u}_{h}\right\|_{h}, \quad\left\|p-p_{h}\right\|_{0, \Omega_{o}} \leq C h^{k-2}\left\|p-p_{h}\right\|_{h},
$$

and by virtue of Lemma 3 once again, the proof is finished.

\subsection{Newton method}

We present the following iterative Newton scheme for the numerical approximation of the nonlinear problem (3). Given an approximation $\left(\boldsymbol{u}_{h}^{r}, p_{h}^{r}\right)$, for $r=0, \ldots$ until convergence, solve: find $\left(\boldsymbol{u}_{h}^{r+1}, p_{h}^{r+1}\right)$ such that

$$
\begin{aligned}
a_{h}\left(\boldsymbol{u}_{h}^{r+1}, \boldsymbol{v}_{h}\right)+c_{h}\left(\boldsymbol{u}_{h}^{r+1}, \boldsymbol{v}_{h}\right)+b_{h}\left(p_{h}^{r+1}, \boldsymbol{v}_{h}\right)=F^{r}\left(\boldsymbol{v}_{h}\right) & \text { for all } \boldsymbol{v}_{h} \in \mathcal{V}_{h} \\
b_{h}\left(q_{h}, \boldsymbol{u}_{h}^{r+1}\right)+d_{h}\left(p_{h}^{r+1}, q_{h}\right)=G^{r}\left(q_{h}\right) & \text { for all } q_{h} \in Q_{h},
\end{aligned}
$$


where the notation $F^{r}(\cdot)$ and $G^{r}(\cdot)$ denotes a direct dependence on the solution at step $r$. Notice that the algorithm starts with $\left(\boldsymbol{u}_{h}^{r}, p_{h}^{r}\right):=(\mathbf{0}, 0)$, and it is stopped when the corresponding residual

$$
\varepsilon_{k}=\left\|\boldsymbol{u}_{h}^{r}-\boldsymbol{u}_{h}^{r-1}\right\|_{h}\left\|\boldsymbol{u}_{h}^{r}\right\|_{h}^{-1}+\left\|p_{h}^{r}-p_{h}^{r-1}\right\|_{h}\left\|p_{h}^{r}\right\|_{h}^{-1},
$$

satisfies $\varepsilon_{k}<\epsilon$, for a prescribed tolerance $\epsilon$. Even though (3) and (5) admit a unique solution, the previous Newton scheme may not converge for any arbitrary value of the activation function $\gamma_{\mathbf{f}_{o}}$. As we will confirm in the next section, for some large deformation problems it is required to employ an incremental step method, which consists essentially in moving smoothly deformations and pressure from $(\mathbf{0}, 0)$ to the desired final state by incrementing the activation $\gamma_{\mathbf{f}_{o}}$.

\section{Numerical examples}

This section contains three tests assessing the accuracy and robustness of the proposed discontinuous formulation. For sake of comparisons, we will also present convergence results for classical Taylor-Hood finite element methods. We recall that in our case, the linearized stability of the numerical scheme will imply convergence of the method for the linearized nonlinear elasticity problem [25]. However this is not the case in general. The strategy of combining error estimates derived for the linearized problem with the known convergence of the Newton method (ensured by the differentiability of a nonlinear operator associated to the bilinear form $b(\boldsymbol{u}, p)$, and a compatibility condition, see $[17,26]$ ), would imply convergence of the DG approximation to the solution of (3) only in the case of relatively small errors (see also [7, 24]).

\subsection{Validation and convergence history}

As first example, we consider the unit square domain $\Omega_{0}:=(0,1)^{2}$, we set the Dirichlet datum $\boldsymbol{u}=(x, y)$ on $\Gamma_{\mathrm{D}}=(0,1) \times\{0\}$ and a suitable traction term $\boldsymbol{t}$ on $\Gamma_{\mathrm{N}}=\Omega_{o} \backslash \Gamma_{\mathrm{D}}$ in such a way that the exact solution for (2) is the smooth pair

$$
\boldsymbol{u}=\left(b x+\frac{a}{2} \mathbf{f}_{o}^{y} y^{2}, \frac{y}{b}\right)^{T}, \quad p=\mu a b\left(1+\gamma_{\mathbf{f}_{o}} \mathbf{f}_{o}^{y, 2}\right)^{2}\left(x+\frac{a y^{2}}{2 b}\right) .
$$

This solution satisfies the incompressibility constraint $\operatorname{det} \nabla \boldsymbol{u}=1$. The chosen parameters are $\mu=400, a=1, b=1$. Fibers are aligned with a fixed angle of $\pi / 2$ and sheets follow the $-y$ axis, that is, $\mathbf{f}_{o}=(0,1)^{T}$ and $\mathbf{s}_{o}=(-1,0)^{T}$. The mechanical activation is assumed constant $\gamma_{\mathbf{f}_{o}}=-0.3$. The stabilization factors take the values $\alpha=0.1, \beta=10000, \delta=0.001$. 
The approximate solution (deformation represented on the reference body and pressure field on the deformed configuration) is plotted in Fig. 1.

To assess the convergence of the stabilized method, we make use of relative errors and experimental rates of convergence defined by

$$
\begin{aligned}
e_{m}(\boldsymbol{u}) & :=\frac{\left|\boldsymbol{u}-\boldsymbol{u}_{h}\right|_{k, \Omega_{o}}}{|\boldsymbol{u}|_{k, \Omega_{o}}}, e_{0}(p):=\frac{\left\|p-p_{h}\right\|_{0, \Omega_{o}}}{\|p\|_{0, \Omega_{o}}}, \\
E(\boldsymbol{u}) & :=\frac{\left\|\boldsymbol{u}-\boldsymbol{u}_{h}\right\|_{h}}{\|\boldsymbol{u}\|_{h}}, E(p):=\frac{\left\|p-p_{h}\right\|_{h}}{\|p\|_{h}}, \\
r_{m}(\boldsymbol{u}) & :=\frac{\log \left(e_{m}(\boldsymbol{u}) / \hat{e}_{m}(\boldsymbol{u})\right)}{\log (h / \hat{h})}, r_{0}(p):=\frac{\log \left(e_{0}(p) / \hat{e}_{0}(p)\right)}{\log (h / \hat{h})}, \\
R(\boldsymbol{u}) & :=\frac{\log (E(\boldsymbol{u}) / \hat{E}(\boldsymbol{u}))}{\log (h / \hat{h})}, R(p):=\frac{\log (E(p) / \hat{E}(p))}{\log (h / \hat{h})},
\end{aligned}
$$

$m \in\{0,1\}$, where $e_{m}$ and $\hat{e}_{m}$ are errors obtained for two consecutive meshes of sizes $h$ and $\hat{h}$. We consider a sequence of successively uniformly refined triangular meshes of 1, 9, 49, 225 and 961 interior nodes. The Newton algorithm is stopped when the residual attains the desired tolerance of 1e-7. On each iteration, the (nonsymmetric) linear systems are solved with the UMFPACK method. Table 1 along with Fig. 2 display the error history for this test problem using a standard continuous Galerkin Taylor-Hood formulation and two stabilized DG formulations using continuous and discontinuous pressures. In the log-log scale we plot the relative errors versus the number of nodes for each discretization. We can see a similar overall behavior for all schemes: a convergence rate of $h^{3}$ is observed for the $L^{2}$-norm of deformations and of $h^{2}$ for the $L^{2}$-norm of the pressure and $H^{1}$-seminorm of the deformation; all these well in accordance with our theoretical findings.
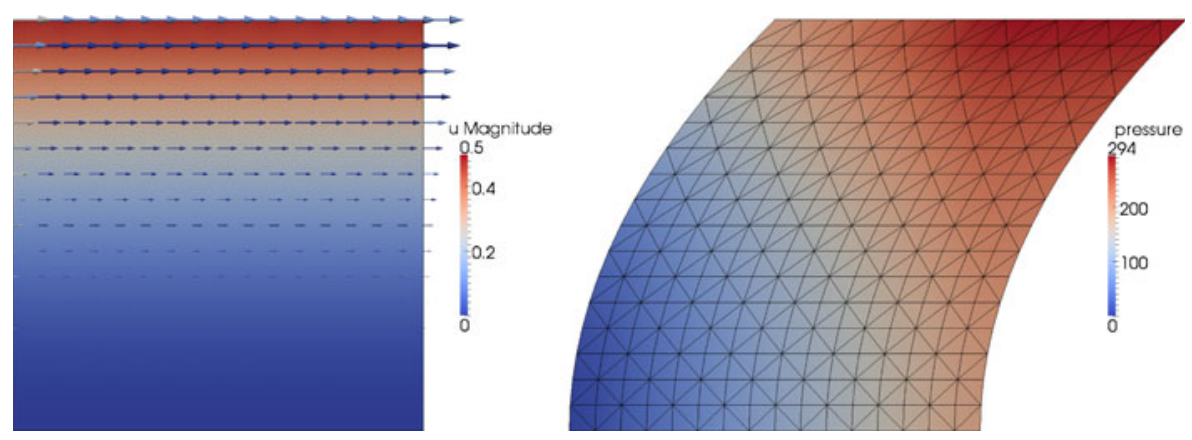

Fig. 1 Test 1: Deformation field on the reference configuration (left) and pressure distribution on the corresponding deformed domain (right). Solution obtained on a uniform mesh of 512 elements, using a stabilized $\mathbb{P}_{\text {disc }}^{2}-\mathbb{P}^{1}$ method 


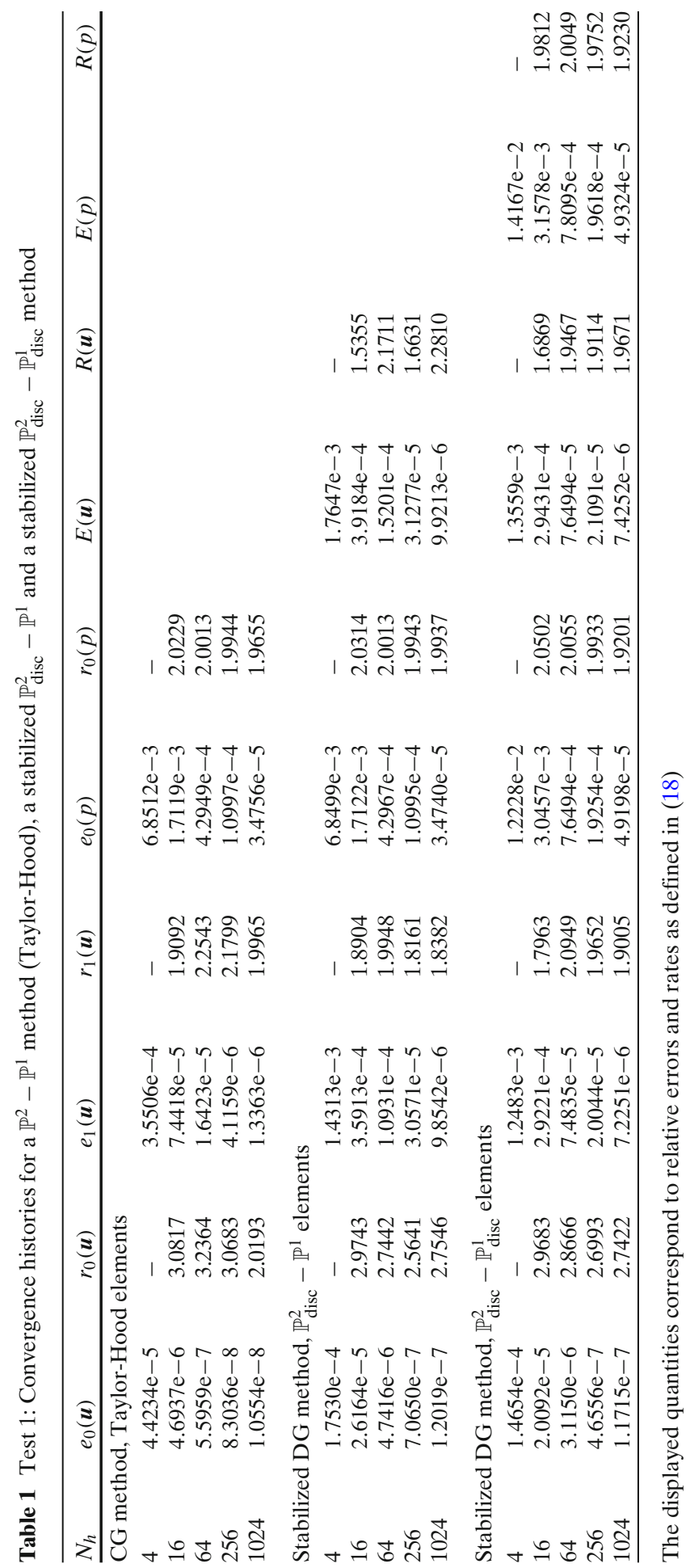



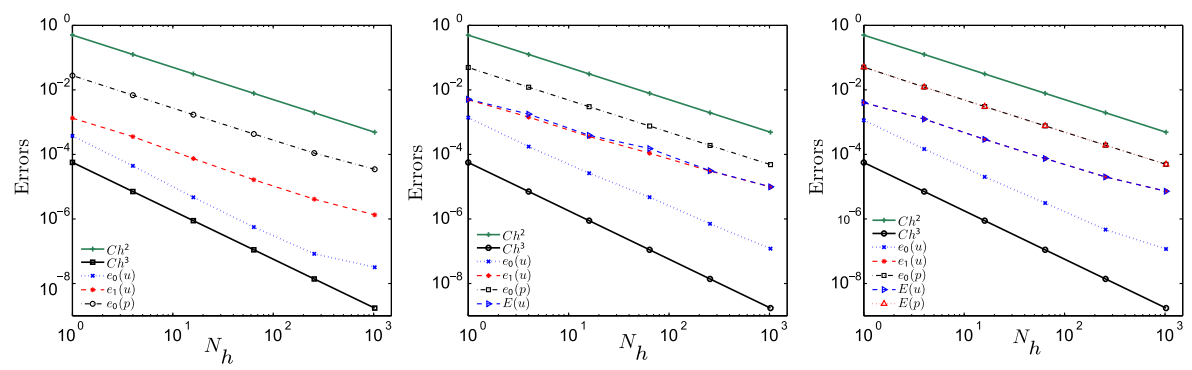

Fig. 2 Test 1: Convergence behavior of a Taylor-Hood method (left) and stabilized DG methods of type $\mathbb{P}_{\text {disc }}^{2}-\mathbb{P}^{1}$ and $\mathbb{P}_{\text {disc }}^{2}-\mathbb{P}_{\text {disc }}^{1}$ (middle and right, respectively). The displayed quantities correspond to relative errors as defined in (18)

\subsection{Active contraction in a $2 \mathrm{D}$ slab}

In the second example we consider the simulation of the active contraction of a neo-Hookean material with elastic modulus $\mu=400$ and fibers and sheets aligned with $\mathbf{f}_{o}=(0,1)^{T}$ and $\mathbf{s}_{o}=(-1,0)^{T}$, respectively. The activation function is no longer constant, but we choose $\gamma_{\mathbf{f}_{o}}=0.3 \exp \left(4[y-0.25]^{2}\right)$. On the base of the square we impose $\boldsymbol{u}=\mathbf{0}$. With a mesh of 625 interior nodes, Taylor-Hood elements exhibit reverse triangle deformation, and volumetric locking. With $\mathbb{P}_{\text {disc }}^{2}-\mathbb{P}^{1}$ elements we obtained correct deformations, but the pressure presented spurious oscillations near the lines $x=0$ and $x=1$. The best results were obtained with a stabilized $\mathbb{P}_{\text {disc }}^{2}-\mathbb{P}_{\text {disc }}^{1}$ method, for which the numerical solution is depicted in Fig. 3; we observed no oscillations in the pressure field, and preservation of the material's volume.

\subsection{Active contraction in a cube}

Finally we perform a 3D test involving an active neo-Hookean 3D domain with elastic modulus $\mu=400$ and fibers, sheets and transversal directions $\mathbf{f}_{o}=$
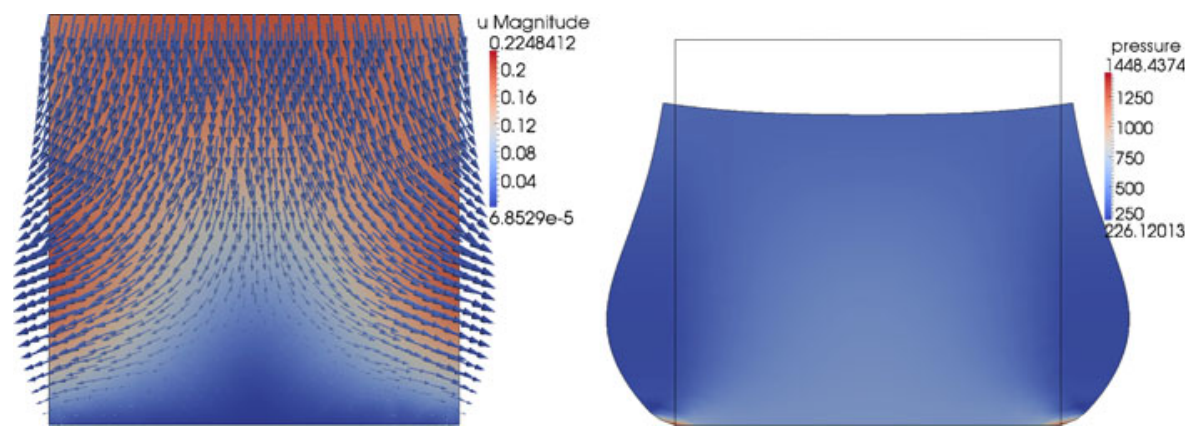

Fig. 3 Test 2: Deformation field on the reference configuration (left) and pressure distribution on the corresponding deformed domain (right). Solution obtained using a stabilized $\mathbb{P}_{\text {disc }}^{2}-\mathbb{P}_{\text {disc }}^{1}$ method 

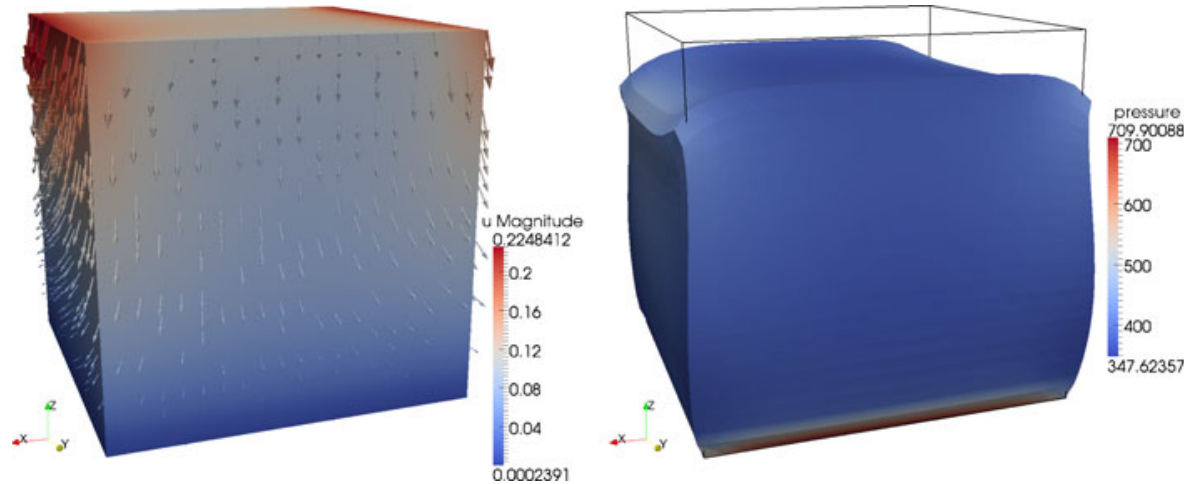

Fig. 4 Test 3: Deformation field on the reference configuration (left) and pressure distribution on the corresponding deformed domain (right). Solution obtained using a stabilized $\mathbb{P}^{2}-\mathbb{P}_{\text {disc }}^{1}$ method

$(0,0,1)^{T}, \mathbf{s}_{o}=(0,1,0)^{T}$, and $\mathbf{n}_{o}=(-1,0,0)^{T}$, respectively. The mechanical activation is $\gamma_{\mathbf{f}_{o}}=0.3 \exp \left(4[z-0.35]^{2}\right)$. On the bottom of the unitary cube we assume $\boldsymbol{u}=\mathbf{0}$. The solution, corresponding to a volume-preserving contraction in the direction of $\mathbf{f}_{o}$, is plotted in Fig. 4. Even if we do not claim that our DG method is less expensive than a comparable continuous FEM, we can state that using continuous finite elements, we were not able to achieve convergence of the Newton method without using a homotopy (or incremental load) method, that is, starting with a small magnitude of $\gamma_{\mathbf{f}_{o}}$ (namely, $\gamma_{\mathbf{f}_{o}}=-0.005 \exp (4[z-$ $\left.0.35]^{2}\right)$ ) and increasing it progressively until the desired value was achieved. With discontinuous formulations, we found that such strategy was no longer needed.

\section{Concluding remarks}

In this paper, an interior penalty stabilized discontinuous Galerkin method for a nonlinear elasticity problem under the assumption of material incompressibility has been proposed. The stability of the numerical scheme has been addressed in detail, and optimal error estimates for deformations and pressure in different norms have been derived, and confirmed by several numerical experiments in two and three spatial dimensions. We stress that in contrast to the behavior observed with continuous finite elements, we do not require any kind of incremental load technique, and the deformations are well resolved at a reasonable computational cost.

The present development was strongly motivated by the study of active deformations in the cardiac tissue as presented in e.g [23], and some already envisaged extensions include a convergence analysis for a DG formulation applied to Holzapfel-Ogden passive materials [13], a posteriori error analysis 
as in [28], and the design of robust preconditioners to speed-up the solution of the involved unsymmetric mixed formulations.

Acknowledgements The authors thank the stimulating discussions with Benjamin Stamm (Berkeley) on the subject of this paper. In addition, A. Quarteroni and R. Ruiz-Baier are financially supported by the European Research Council through the advanced grant "Mathcard, Mathematical Modelling and Simulation of the Cardiovascular System”, ERC-2008-AdG 227058.

\section{References}

1. Arnold, D.N., Brezzi, F., Cockburn, B., Marini, D.: Unified analysis of discontinuous Galerkin methods for elliptic problems. SIAM J. Numer. Anal. 39, 1749-1779 (2002)

2. Auricchio, F., Beirão de Veiga, L., Lovadina, C., Reali, A.: The importance of the exact satisfaction of the incompressibility constraint in nonlinear elasticity: mixed FEMs versus NURBS-based approximations. Comput. Methods Appl. Mech. Eng. 199, 314-323 (2010)

3. Babuška, I.: The finite element method with penalty. Math. Comput. 27, 221-228 (1973)

4. Barrientos, M., Gatica, G.N., Stephan, E.P.: A mixed finite element method for nonlinear elasticity: two-fold saddle point approach and a posteriori error estimate. Numer. Math. 91, 197-222 (2002)

5. Braess, D., Ming, P.: A finite element method for nearly incompressible elasticity problems. Math. Comput. 74, 25-52 (2005)

6. Brezzi, F., Fortin, M.: Mixed and Hybrid Finite Element Methods. Springer, New York (1991)

7. Brink, U., Stein, E.: A posteriori error estimation in large-strain elasticity using equilibrated local Neumann problems. Comput. Methods Appl. Mech. Eng. 161, 77-101 (1998)

8. Cockburn, B., Karniadakis, G.E., Shu, C.W. (eds.): Discontinuous Galerkin Methods. Theory, Computation and Applications, vol. 11. Springer (2000)

9. Ern, A., Guermond, J.L.: Eléments finis: théorie, applications, mise en oeuvre. Springer, Paris (2002)

10. Gatica, G.N., Gatica, L.F., Stephan, E.P.: A dual-mixed finite element method for nonlinear incompressible elasticity with mixed boundary conditions. Comput. Methods Appl. Mech. Eng. 196, 3348-3369 (2007)

11. Girault, V., Raviart, P.A.: Finite Element Methods for Navier-Stokes Equations. Springer, Berlin (1986)

12. Hansbo, P., Larson, M.G.: Discontinuous Galerkin methods for incompressible and nearly incompressible elasticity by Nitsche's method. Comput. Methods Appl. Mech. Eng. 191, 36693750 (2002)

13. Holzapfel, G.A., Ogden, R.W.: Constitutive modelling of passive myocardium: a structurally based framework for material characterization. Philos. Trans. R. Soc. Lond., A 367, 3445-3475 (2009)

14. Humphrey, J.D.: Cardiovascular Solid Mechanics. Springer, Berlin (2002)

15. Lamichhane, B.P.: A mixed finite element method for non-linear and nearly incompressible elasticity based on biorthogonal systems. Int. J. Numer. Methods Eng. 79, 870-886 (2009)

16. Lazarov, R., Ye, X.: Stabilized discontinuous finite element approximations for Stokes equations. J. Comput. Appl. Math. 198, 236-252 (2007)

17. Le Tallec, P.: Existence and approximation results for nonlinear mixed problems: application to incompressible finite elasticity. Numer. Math. 38, 365-382 (1982)

18. Lew, A., Negri, M.: Optimal convergence of a discontinuous-Galerkin-based immersed boundary method. ESAIM: Math. Model. Numer. Anal. 45, 651-674 (2011)

19. Nobile, F., Quarteroni, A., Ruiz-Baier, R.: An active strain electromechanical model for cardiac tissue. Int. J. Numer. Methods Biomed. Engrg. 28, 52-71 (2012)

20. Noels, L., Radovitzky, R.: A general discontinuous Galerkin method for finite hyperelasticity. Formulation and numerical applications. Int. J. Numer. Methods Eng. 68, 64-97 (2006)

21. Ortner, C., Süli, E.: Discontinuous Galerkin finite element approximation of nonlinear secondorder elliptic and hyperbolic systems. SIAM J. Numer. Anal. 45, 1370-1397 (2007) 
22. Quarteroni, A., Valli, A.: Numerical Approximation of Partial Differential Equations. Springer, Berlin (1997)

23. Rossi, S., Ruiz-Baier, R., Pavarino, L.F., Quarteroni, A.: Orthotropic active strain models for the numerical simulation of cardiac biomechanics. Int. J. Numer. Methods Biomed. Engrg. 28, 761-788 (2012)

24. Stein, E., Seifert, B., Ohnimus, S., Carstensen, C.: Adaptive finite element analysis of geometrically non-linear plates and shells, especially buckling. Int. J. Numer. Methods Eng. 37, 2631-2655 (1994)

25. Ten Eyck, A., Lew, A.: Discontinuous Galerkin methods for nonlinear elasticity. Int. J. Numer. Methods Eng. 67, 1204-1243 (2006)

26. Valent, T.: Boundary Value Problems of Finite Elasticity: Local Theorems on Existence, Uniqueness, and Analytic Dependence on Data. Springer (1988)

27. Whiteley, J.P.: Discontinuous Galerkin finite element methods for incompressible non-linear elasticity. Comput. Methods Appl. Mech. Eng. 198, 3464-3478 (2009)

28. Whiteley, J.P., Tavener, S.J.: Error estimation and adaptivity for incompressible, nonlinear (hyper) elasticity. OCCAM preprint 12/75 